$$
.616991: 6189
$$

THE RHEUMATIC DISEASE OF CHILDHOOD.

Being a Thesis for the Degree of M.D., University of Edinburgh.

$$
\text { by }
$$

\title{
PETER CRERAR
}

$$
M \cdot B \cdot, C \cdot M \text {. }
$$

April 1907.

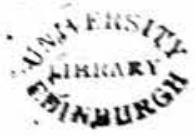




\section{THE RHEUMATIC DISEASE OF CHILDHOOD.}

Acute rheumatism or rheumatic fever as it occurs in childhood differs very much from the same condition as we meet with in the adult. Before reviewing the condition it will be well to consider the views as to the cause of the condition known as Rheumatic Fever.

\section{ETIOLOGY.}

Of the many views advanced as to the cause of the condition - the one that has survived to the prosent day is that Rheumatic Fever is an infectious disease and of bacterial origin.

Since Manele in 1887 foreshadowed its bacterial relation many observers have at various times claimed to have discoverod organims in relation to the disease - Popoff, Achalme, Sahli,Goldschuder, Dana, Widal, Bezancon, Theroloix, Triboulet, Apert, Westphal, Wasserman, Meyer, Menzer, Poynton, Paine, Beaton, Walker, Shaw and Beattie. It seems, however, that one can put aside the different organisms described by the above observers as casual - except the diplococcus of Wasserman described in 1899, and that which is probably the same organism and known as the Micrococcus Rheumaticus, and described by Poynton, Paine, Walker, Shaw and Beattie. 
Poynton and Paine in 1900 isolated a diplococcus from eighteen cases of rheumatic fever. This organism was obtained from the blood-joints and heart valves. It has been cultivated outside the body and when inoculated into rabbits and monkeys produced polyarthritis, endocarditis and other manifestations of rhoumatic fever and has again been isolated from the lesions in these animals. Beaton and Ainley Walker, and again later Poynton \& Paine got sinilar results. Beattie in 1904 obtained an organism from the inflamed patches on the synovial membrane of a rheumatic fever patient and on inoculation in rabbits with it produced polyarthritis, endocarditis and chorea. He could not show distinction, however, between it and other streptococci. Later, in 1906, Beattie in a further contribution gives the results of his experiments. From these he stated that the coccus isolated was in its morphological characters and staining reactions indistinguishable from some strains of streptococcus pyogenes. He was impressed, however, by the vitality of the organism, constant subcultures being necessary to keep the streptococci alive, while after several months quite active subcultures could be obtained of the special organism - the micrococcus rheumaticus. He carried out experiments with twelve strains of streptococci got from various 
sources, and three of the special rheumatic organiam, in all the streptococci inoculations the arthritis was purulent and in the single experiment with endocarditis there was ulceration of the valves and the organisms were invading the adjacent heart muscle, pyaemic abscesses occurred in the kidney and dorsal vertebrae, while in the cases inoculated with the special organism the arthritis was non purulent. Fritz Meyer asserts of the diplococcus isolated by him, which in appearance and relation does not differ from the organism isolated by Wasserman, and Poynton and Paine that one of the characteristics by which it may be distinguished from ordinary streptococci is its greater haemolytic action. Other observers have disputed the claim of specificity to the micrococcus rheumaticus on the ground that the claim rests on the results of inoculations alone and asserting that they have produced the lesions of rheumatic fever - arthritis and endocarditis with other varieties of micrococci. Menzer in 1902, Glaser, Harris and Cole, all cleim to have produced similar lesions with different organisms. We thus have the two views:-

(1) The bacteriologic view, that the disease is a specific infection, due to a specific micro-organism. 
(2) The clinical view, that the disease is not due to a specific infection but that it is a particular reaction of the body to a wide variety of organisms.

Cole being one of the chief supporters of the clinical view, Beattie in criticising his conclusions, points out that Cole, while working with an organism which he admits might be the micrococcus rheumaticus, in no case was the exudate purulent, but resembled that obtainod by Beattie, while cole in his experiments with other streptococci got usually a purulent exudate or turbid fluid in the joints, while Cole's inoculations frequently resulted in the death of the animals from septicaemia - in those inoculated with the special organism, by Poynton and Paine and Beattie the animals usually lived.

Beattie concludes that Coles experiments merely show that purulent arthritis in rabbits is a frequent manifestation of streptococci injected intravenously, but that they fail to prove that other varieties of streptococci produce the same results in anjmals as the micrococcus rheumaticus.

Some of those holding the clinical view such as Sahli and Singer regard rheumatic fever as an attenuated pyaemia. In reply to this' Poynton and Paine point out that the organism is very virulent and is found associated with very severe forms of ulcerative endocarditis. It never produces pus nor a 
picture resembling that of streptococcus - septicaemia. Beattie also replies to this and directs attention to the very severe symptoms and often rapidy fatal issue of rheumatic fever, and shows that in pyaemia and especially where the organism is not very active pus formation is the common result, whereas in acute rheumatism pus formation is the exception. McRae suggests that since the organisms described were usually found in fatal cases they might mean simply a terminal infection. In reply to this Beattie points out that the organism has been isolated during life, and that in a terminal infection the organism would be general and found in the blood, whereas cultured tubes inoculated from the blood remained sterile, cultures of the organisms being got from isolated areas in the synovial membranes only. Poynton in 1904 in cultures from the blood and pericardial fluid from a fatal case of rheumatism with chorea got negative results and emphasised the fact that the organisms thrive best in the local lesions, and do not thrive in the blood, although occasionally present.

Those who support the clinical view need further evidence to show that with a variety of organisms of the streptococcus group, they can produce with the same constancy and accuracy the lesions and clinical course of rheumatic fever, as that seen in 
the experiments with the organism derived from rheumatic patients, although it may be allowed that the organisms may produce arthritis and endocarditis. While if an organism can claim to be specific from the results of inoculation in animals alone, then the weight of evidence is in favour of the micrococcus rheumaticus being the specific cause.

If we think of the disease as we meet with it clinically, then we must admit that although rheumatism has many analogies to an infection by pyogenic cocci, that considering the sameness of the cases and the close similarity of all the cases to each other, and the constancy with which the same lesions are produced, the course of the disease, the range of temperature, the family history etc., the reaction to be specific and due to a specific cause, and not due to a variety of organisms, where we should be likely to have very varied reactions according to the individual attacked and to the multiple character of the organisms with never the same periodical recurrences or cylic course that one generally gets in rheumatic disease and most probably with pus formation which never occurs in the rheumatic condition. Thus one feels that the disease, as seen by the physician, is alone sufficient justification for regarding rheumatic fever as a definite disease - a specific infection - and due 
to the micrococcus rheumaticus of poynton, Paine, Beattie and others.

General characteristics of the rheumatic disease of childhood as compared with that of adults.

Acute rheumatism as we know it in the adult, comes on rather abruptly, with great prostration, acute and painful swelling in several joints, with sour, profuse, acid smelling perspirations - the affected person being unable to move in bed, and practically fixed with pain, with the temperature generally $102^{\circ}-104^{\circ}$. Not so in the child, where the first thing that strikes one is the insidious nature of the disease and the comparative mildness of the joint affection. In fact, there may be entire absence of the arthritis. Then other phases of the rheumatic state become more prominent. Cardiac manifestations are much more frequent, and may be the only manifestations of the disease. In fact, one finds the cardiac manifestation alone more frequent than one finds arthritis alone, and we may have endocarditis or pericarditis as the primary manifestation. Thus in the child, such conditions as endocarditis, pericarditis, pleurisy, tonsillitis, chorea, and exudative erythemata and subcutaneous nodules, which used to be classed as complications following arthritis, are now known to 
be true manifestations of the disease. This was well brought out in the classic paper of Barlow in 1883 in introducing the discussion on the subject at the annual meeting of the British Medical Association, and in the Harveian Lecture by Cheadle in 1889. Cheadle emphasized the insignificance of the joint affection and the greater prominence and frequency of other phases, and made up a rheumatic series, in which he included with the arthritis, endocarditis, pericarditis, pleurisy, tonsillitis, exudative erythemata, chorea and subcutaneous nodules. To this series I shall add cardiac dilatation. The different members of the series may occur singly and independently of each other or we may have several of the series occurring close together - or again we may have each of the members of the series occurring in one case, but spread over several years, bearing out what cheadle says, that the rheumatic history of a child may be that of its childhood. Usually we have arthritis first alone, or with endocarditis, pericarditis and chorea in one attack, or arthritis with endocarditis, endocarditis alone, or endocarditis with chorea. In fact, we may have many different orders of sequence either in the same attack or at longer intervals of time.

During the last two years I have had several cases, showing each phase of the series, and as the 
children with their families have been known to me for ten years at least, I have been struck with the spread-out character of the disease, as seen in childhood, with the evidence of heredity and the influence of sex.

I now give some of the cases, all within the last two years.

The first three cases were all members of one family and give a history of rheumatism, both on the father and mother's side, the mother having had rheumatic fever, and the father's sister having had rheumatism, and her own boy dying from the disease.

Horace Adshead: 10 years of age. Has had tonsillitis, twice or thrice a year in the last four years - pleurisy twice, and broncho-pneumonia twice. He has always been a pale looking boy. Has a good home and well cared for. In November of 1905 his father brought him to me. He complained of pain behind the knee and in the groin, and walked on tip-toe, limping. There was no pain or swelling in any of the joints. He was anaemic. on examining heart, one found the action rapid, and there was some dyspnoea: temperature was normal. I judged the condition as rheumatic and ordered rest in bed, with salicylates. He was well in a week.

In November of 1906 I saw him again. A fortnight before I saw him he had sore throat and was 
kept from school for one week. After one week at school he took ill again. I found him in bed, with pain in and slight swelling of the left knee, temperature $101^{\circ}$, rapid action of heart with short breathing and distinct signs of endocarditis. With salicylates the pain in the joint passed away in two or three days. The endocarditis, however, got worse and a loud murmur was audible. The salicylates controlled the temperature, but on the least breaking it off, the temperature rose again and the heart's action became again very rapid. About fourteen days from the beginning of the attack, signs of pericarditis appeared, much pain in the praeCordial region with dread of breathing. Increasing the salicylates relieved the pain in this as well. The condition gradually improved, but in the fourth week chorea set in and here again on increasing the salicylates the movements quietened down, leaving him with loss of voice and loss of power in the neck, arms and legs, for fourteen days. He gradually improved and after three months in bed was allowed to get up. His heart now shows evidence of the destructive nature of the disease and a loud murmur is heard of mitral origin.

Ernest Adshead: aged 8 years, was brought to me in November 1905, a fortnight after his brother. He had the same limping symptom and was unable to 
put the right heel to the ground, the hamstring tendons were stiff and painful. He was anaemic. His heart was rapid, no temperature. One week's rest with salicylates and he appeared well. He is still anaemic and thin, but so far has shown no further manifestation of the disease.

Eli Adshead: aged seven years. Was brought to me in December 1905, because of looking so pale and being short of breath. While walking to my house he was suddenly seized with pain behind the right knee and like his brothers was unable to put his heel to the ground. He was very anaemic, short of breath and looked very ill. Temperature $101^{\circ}$ and distinct signs of endocarditis. He was sent home to bed and seemed to be progressing favoutably, but on the tenth day he died suddenly; the heart was greatly dilated.

Having known these boys throughout their childhood, nothing impressed me more than their continued pale appearance, and in the light of the rheumatic manifestations of the last two years, I feel that in all three a mild infection was going on, even without rise of temperature.

Horace, thus, has history of tonsillitis, then tonsillitis with arthritis, endocarditis, pericarditis, chorea. 
Emest, the anaemia with rheumatic inflammation of the periarticular tissues of knee - not amounting to actual arthritis.

Eli, endocarditis, the primary manifestation, no arthritis.

Frances Buckley: 14. Tonsillitis, arthritis, endocarditis. Mother had rheumatic fever, with now a mitral murmur. First saw this case in August 1906, ten days after she had sore throat. Here the arthritis was more approaching the adult type, the joints being more swollen and painful than usual in a child. I saw her a month ago, when the murmur was nearly gone.

May Gilbert: Seen in February 1907. She was sitting by the fire, looked anaemic and distressed. Distinct endocarditis; temperature $101^{\circ}$. She got well in three weeks. Still looks rather anaemic, no murmur. Here there was no arthritis. Father, mother and brother have had rhoumatic fever.

Mabel Bouring: Seen in January 1907. 14 years of age. Arthritis, with endocarditis and at present time chorea, with subcutaneous nodules. A year ago she had arthritis with chorea following. I did not see her then, but think probably there was 
endocarditis. This case, I think, bears out Cheadle's views as to the grave significance of nodules, as now there is a subacute smouldering endocarditis going on and after nearly four months the condition does not improve. The temperature 970 has been so for six weeks.

Winnie Hirst: aged 13, seen beginning of April of this year. This child was sent from Burton-on-Trent for change of air. She was seized with rheumatism last November, arthritis with endocarditis. I saw her once, as she had suddenly become worse, with attacks of sickness. There was endocarditis, chorea and subcutaneous nodules. Six hours after I saw her she died suddenly on raising herself up in bed, evidently dilatation following the straining associated with sickness. In this case again, do we find nodules, a long attack from November 1906 to April 1907, with cardiac failure at the end.

Doris stevenson: 13 years of age. This case I have seen to-day; found her in bed with painful and swollen left ankle, temperature 1020; here, again, approaching the adult type. She had tonsillitis a fortnight ago . Looks very anaemic. Her mother drew my attention to the fact that I noted her as being very anaemic a year ago, while 
I was seeing another member of the family. Her brother had rheumatic fever two years ago. He is 19 years of age now and has a mitral murmur which I detected ten years ago. He was never laid up and his mother remarked that he had only suffered from growing pains. He evidently had rheumatism as a child, which was not noticed at the time.

Gertrude Hall: aged 13 years, seen in February 1907. Mild arthritis, temperature 1010; with salicylates and rest soon recovered. Her sister living at Wolverhampton was then suffering from chorea.

George Harvey: 11 years. Tonsillitis, arthritis, purpuric eruption. Father and mother both rheumatic.

These cases all bear out Cheadle's observations - the insidious nature of the disease; the mildness of the arthritis and the prominence especially of cardiac affection; the occurrence of the different phases in the same attack or at different times and in different attacks, and thus being spread out through a childhood; also show how the arthritis becomes more of the adult type after 13 years of age. They also show the predominance of cases in girls, between 11 and 15 years of age, thus agreeing with the statistics generally. 


\section{Influence of sex:}

If we take males and females of all ages together, we find articular rheumatism is somewhat more common in the former. The statistics of the Collective Investigation Committee of the British Medical Association yield in 655 cases, 375 males to 279 females. If we take up to the age of 20 only, then there is a difference. The Collective Investigation statistics give 108 males to 120 females. After 20 the males are in excess; viz. 263 to 158. If, however, we take periods of five years from one to twenty years, we find in the first five boys are in excess 5 to 1 . Between five and ten years, they become nearly equal, 15 boys to 14 girls; the next period from 11 to 15 years shows a distinct remarkable change - girls predominate in the proportion of two to one -47 girls to 25 boys; then up to twenty years the greater liability of girls decreases, so at the close of this period males again predominate, 76 males to 67 females.

\section{Heredity:}

In all of my cases there was distinct history of rheumatism in one or both parents. Dr Goodhart taking children alone found satisfactory evidence of rheumatic affection in 41 out of 69 or $57 \%$. 
I think with careful investigation and especially in private practice where one can get better and more reliable information the percentage would be much higher. In all my rheumatic cases both in children and adults during the last 13 years, I always proved heredity .

In considering the various members of the series in detail, and beginning with the Arthritis we note, as has been already mentioned, its comparative mildness and insignificance and the possibility of its being absent altogether. We rarely get the painful swollen joints one gets in the adult, although the nearer puberty we get, the nearer do we seem to get to the adult type, and see it more frequently. As a rule the arthritis is limited to one or at most two joints, is not very painful and rarely lasts longer than 48 hours. It would seem that in the child the periarticular tissues are more liable to be affected than the synovial membranes. The rheumatic virus in the child acting more on fibrous structures, this distinction passing away with the adult type. Then since we have the arthritis so mild and evanescent, one rarely gets a temperature above $101^{\circ}$ or $102^{\circ}$. As a rule the temperature is above 1000 . More often, in fact, in the severe forms in chilahood the temperature may never be higher than $99^{\circ}$ to $100^{\circ}$ and yet 
the disease run a rapid and fatal course. The mildness of the arthritis is misleading and children are not taken to their medical man, although a distastrous endocarditis may be developing.

\section{Anaemia:}

This is one of the most important symptoms and when seen in a child should make us think of rheumatism, and fix our attention on the cardiac condition. In no other disease except diphtheria does one get such marked anaemia in children. All my cases were markedly anaemic, although the greater number belonged to good homes, and were well cared for, all living in the country, while the non-rheumatic members were not anaemic. With cardiac affection the anaemia progressively deepens and especially in pericarditis cases the anaemia is profound.

\section{Tonsilitis:}

This also is a distinct manifestation of the rheumatic state. Some, such as Snyder, hardly admit this in to the series, since tonsillitis is so often associated with streptococci infection, but the rheumatic sore throat is not like the septic throat where one gets a high range of temperature, muco-pus and enlarged glands. Here rather do we get a diffuse redness, not confined to the 
tonsils, and the condition lasts rarely longer than two or three days, and soon improves under salicylate treatment. Trousseau recognised a rheumatic sore throat. The Collective Investigation Committee give a percentage of 24. Dr Archibald Garrod and Mr Cooke found percentage of cases of tonsillitis with rheumatic history exactly the same as percentage of articular rheumatism with rheumatic history - 35\%.

Mr Connell, in Recoras of Vanderbilt Clinic, 500 cases of rheumatism, tonsillitis $35 \%$. Montreal Hospital Records 13\%. Gurich reports 17 cases of rheumatism, tonsillitis in 13 .

The tonsil is probably the means of entry for the rheumatic virus just as it is now recognised to be in scarlet fever, diphtheria and probably in pneumonia. The recent experimental work of Fritz Meyer in Germany and of Frissell in the Presbyterian Hospital of New York in which organisms were isolated from cultures taken from the throats of rheumatic patients which in a series of inoculation experiments always gave rise to an arthritis, point to the same pathway for the rheumatic infection.

Meyer in 1901, in five caser found streptococci in sore throat without rheumatism. These, when inoculated in guinea pigs always produced subcutaneous abscesses while the streptococci from the throats in rheumatic cases when inoculated never 
caused suppuration but caused polyarthritis. Menzer in 1902 confimed Meyer's work.

Ross and Bloeg have called attention to the relationship and in 1789 Eyerlen in his "Malina Rheumatica and Tonsillas Deposita" recognised a relation between rheumatism and the tonsils (Dumn). Several of my cases had a history of tonsillitis preceding the arthritic attack, but in none did the tonsillitis and arthritis occur at the same time, at least one week elapsed before development of the arthritis. Some observers, however, have noted the occurrence of tonsillitis at the same time as the arthritis and in others it seems to have come after the arthritis.

\section{Exudative Erythemata:}

In a considerable number of cases this group of skin affections appears to have a septic or toxin origin and although accompanied by pyrexia and articular pain cannot be regarded as having a rheumatic source. In others, however, the history and course of the infection point strongly to a rheumatic origin. The various forms in which the emptions appear are Erythema marginatum, Erythema papulatum, Erythema Nodosum. Dr Barlow and Dr Warner found in twenty seven patients with rheumatic nodules, eight had Erythema papulatum or marginatum, one urticaria and one purpura. The erythema may occur at any 
20.

point in the rheumatic series but it is usually associated with other evidences of rheumatic disturbance. On the whole, we may say that the erythemata are in some cases manifestations of the rheumatic state, but one could hardly diagnose the condition as rheumatic on the appearance of these alone, while occurring with, or following other members of the series, the probability is we could then class them as rhoumatic. So with purpura: although it is generally accepted that there is true rheumatic purpura, without the arthritis, one could hardly term it truly rheumatic, or unless there was a family history of rheumatism. It is difficult to tell when we have purpura from some other toxic cause and when rheumatic, and in the absence of other rheumatic symptoms, one would not be justified in so classifying the condition. Still all are agreed that purpura does occur in the rhoumatic state. I had one case in which the symptoms and family history were sufficiently strong to justify the term rheumatic purpura.

\section{Chorea:}

All are probably agreed on the close relationship between rheumatism and chorea, but there has been much discussion as to the closeness. Dr Barlow in his introduction to the discussion of Rheumatism at the meeting of the British Medical 
Association in 1883, observed that there is no other general disease or fever of childhood with which chorea has any such association, with the single exception of scarlet fever, and that in far less degree, and scarlet fever is the one fever especially associated with acute rheumatism.

"With the exception of rheumatic fever, there is no intimate relationship between chorea and the acute diseases incident to childhood." (Osler). Roger, writing in 1866, regarded articular rheumatisin, chorea and endocarditis as different names applied to one and the same pathological condition. He holds that all chorea is rheumatic. Stahl, Copland, Babington, Todd, Sée, Roger, Simon, Cadet, de Gassecourt all taught rheumatic origin. French authors as a rule have urged the rheumatic causation of chorea. Cheadle believed chorea might have other causes as well as rheumatism, but was convinced that rheumatism was the most common and potent factor. Statistics have been collected by various observers, bearing on the relationship.

Osler, 554 cases: in $15.5 \%$ there was a history of rhoumatism in the family. In 88 cases $15.8 \%$ there was a history of articular swelling acute or subacute. In 33 cases there were pains not associatod with joint trouble, which, if added to those with manifest articular trouble raised the percentage to nearly 21 . 
22.

Tylden found the percentage as high as $72 \%$ in those with a family history of articular affection, and $50 \%$ in those with a personal history.

The British Medical Association Collective Investigation Committee found that rheumatism preceded the chorea in $26 \%$, accompanied or occurred subsequently in $32 \%$. If patients who were the subjects of vague pains were included, the percentage rose to $46 \%$. Townsend gives $21 \%$ Crandall the largest, $54 \%$.

Dr Stephen Mackenzie found 44.76 per cent almost certainly rhoumatic. Dr Barlow finds in 44 out of 73 or 60 per cent, sufficient evidence of rheumatism and points out that the existence of progressive heart disease and the imperfection of the record render it probable that many other cases should be included. The percentage of cases preceded by rheumatism varies with the age of the patient. In children under nine years of age, Sir William Gowers met with one instance only among his cases in which there was a history of previous rheumatism, while between the ages of ten and fifteen he found such a history in more than one fourth. Cheadle found in 84 cases investigated by himself satisfactory evidence of acute rheumatism in the pationt or immediate relatives in 62 , i.e. in 75 per cent. Lees is a staunch supporter and thinks 
nearly all the cases are due to rheumotism, and calls it Central Rheumatism. He thinks with Cheade and others that the mistake is often made of only thinking of rheumatism in the child as represonted by the arthritis without taking into account the other manifestations of rheumatism, such as nodules, and I would add the anaemia. I feel that a constant infection may be going on for years without any other evidence of the rheumatic condition. Chorea itself is often the first of a series of rheumatic attacks. If this be ignored and the relationship of the two diseases be based simply on the precedence of rheumatism, the connection of the two will inevitably be understated. Lees had an investigation made of the amount of subsequent rheumatism occurring within a few years after an attack of chorea in children who had no rheumatism previously. Dr Batten made this for him. He found in 115 cases of chorea that whereas the percentage of previous rheumatism was stated to be only $32 \%$, three years later so many of them had subsequently suffered from rheumatism that the percentage rose to 43.5 , and three years later still it had risen to 53.2 , so that the lapse of six years had increased the percentage by 21\%. Even this was below the truth for after the lapse of six years as many as 38 out of the 115 children could not be in- 
vestigated. If the same proportion of these missing ones had become rheumatic, the percentage would have been increased not by 21 but by 28\%. This added to the cases of previous rheumatism would give a total percentage of $60 \%$. Even these figures, Lees thinks, are too low, since they are based on the idea of rheumatism as represented by joint pain or swelling, while there may be conclusive proof of rheumatism by some other of Cheade's series such as a solitary nodule, or patch of erythema - or a simple dilated heart. Another point in favour of the relationship is, that chorea is much more common in girls, between 10 and 15 years of age, which is also the period in which the rheumatic state predominates in girls - in the ratio of almost two to one. We thus find the clinical evidence of the relationship very strong. In all the cases that have come to my knowledge - seven in all, during the last twelve months - the rheumatic connection has been evident.

When one comes to the pathological evidence, we again refer to the work of poynton and paine. One of the rabbits injected with a culture of their diplococcus obtained by incubating pericardial fluid from a fatal case of rheumatism developed symptoms remarkably resembling chorea. This rabbit was killed and diplococci were found in the lymphatic sheath of the vessels in the pia 
mater and in the endothelial cells of the blood capillaries, penetrating the cortex of the brain. Beattie also got choreic symptoms in rabbit on inoculation with culture of diplococcus from case of rhoumatism. Dana, Apert, Wasserman and others have isolated a diplococcus from the brain in fatal cases of chorea. The embolic theory, supported by Hughlings, Jackson, Tuckwell and Broadbent is not now believed in, since such an opinion finds no support in the findings of morbid anatomy .

Lees calls attention to the investigation of two fatal cases by modern histological methods by Dr Reichardt of Chenmitz which showed small haemorrhages irregularly scattered, with collections of leucocytes, chiefly mononuclear and dilatation of vessels, with perivascular small celled infiltration in many parts of the brain in varying amount. No changes were detected in the ganglion cells, but there were areas of fatty degeneration of nerve fibres; in the spinal cord the parts most affected were the root fibres and the anterior and lateral horns, and the posterior columns; the anterior and lateral tracts were free. Cultures from the fluid of the cerebral ventricles of the first case gave no result, but "staphylococcus aureus" was obtained from the heart blood. In the second case there were streptococci in the cardiac valves and a 
few colonies of staphylococcus albus were obtained from the brain.

Hudovering gives an account of a case in a girl of 16 years, who died of chorea, having had one month earlier an attack of polyarthritis. At the Post-Mortem, bacteriological investigation of the blood and meningeal fluid proved negative, nevertheless the histological findings strongly suggested an infectious origin. Thus there were numerous small haemorrhages widely distributed similar to those seon in Carbonic Acid Intoxication. In the contral nervous system the lesions were chiefly in the medulla and pons and these specially in the motor tracts. The vessels were markedly affected with thickening of the sheath and cellular and oedematous infiltration. In the pyramidal tracts were found curious colloidal formations, more or less closely related to the vessels. He describes the condition as an encephalitis, a low grade ependymitis with leptomeningitis, with changes of a sclerosing type in the pyramidal tracts.

The infectious nature of chorea is now practically accepted. Leube speaks of the probability of a "chemical infectivie nature" and is supported by Reichardt. Hudovering sums up:- 


\section{7 .}

(1) Chorea an infectious disease: the noxa producing their effects via the blood stream.

(2) The colloidal changes occur only in very bad cases.

(3) These when present are characteristic.

(4) The manifestations of chorea are the expression of a direct or indirect stimulation of the pyramidal tracts.

Poynton again tabulates the facts as we have them at present, thus:-

(1) Micro-organisms of the streptococcal group have been isolated from rheumatic fever.

(2) They have been isolated from the cerebrospinal fluid and brain itself in chorea.

(3) They have been demonstrated in the pia mater and brain.

(4) Involuntary் movements of a peculiar type have been recorded by paine and myself and F. Meyer as resulting from intravenous inoculation of rabbits with such micro-organisms.

(5) Identical bacteria are found in other rheumatic lesions in man, and are capable of producing the lesions of rheumatism in animals. 
28.

(6) Chronic leptomeningitis has been noted in chorea by Dana.

(7) The lesions found after death in an acute chorea are such as one could explain on the view of an infection. I mean the minute thrombuses and haemorrhages, occasional embolism, degenerative changes in nerve cells, and perivascular exudations. Poynton holds the view of chorea being an infection of the nervous system comparable to the infection of the nervous system in tuberculous meningitis rather than that of the nervous system in diphtherial paralysis. As regards rheumatic fever and chorea he holds the view that one microorganism is the cause.

In summing up, it seems now to be agreed that chorea is of the nature of an infection, the rheumatic toxin being the cause in the great majority of cases; and this would be found to be more often the cause if other manifestations besides the arthritis were taken into account, and more attenlion given to a study of the earlier and later life history of patients and their families in which the disorder has occurred. Sir Dyce Duckworth's statistics show a family or personal history of rheumatism in $85 \%$ of cases of chorea. At the same time, it must be allowed that other toxines can produce chorea. The older idea of the induction 
of chorea by nervous shock or fright, has but few supporters to-day, and should be discarded. Nevertheless, we must acknowledge a neurotic factor in a true conception of the pathogeny of chorea, and realise a physiological basis in that greater mobility of the nervous system and motor readiness of expression described by Dr sturges and in the more excitable temperament of quite intelligent children and of girls as compared with boys.

\section{Subcutaneous nodules:}

These are now recognised as manifestations of the rheumatic state in the child. They were described as far back as 1868 by Hellier, but Barlow and Wamer were the first to direct attention to their frequency and association with rheumatism. Cheadle laid great stress on their importance and held their presence to affect the prognosis, making it much more grave. I have only noted their presence in two cases, both of which were of an insidious but progressive nature, both in girls, with endocarditis, pericarditis and chorea. One of the cases ended fatally, the other still suffers from chorea, while there seems to be an insidious, subacute progressive endocarditis going on.

These nodules lie under the skin and are connected with fascia or tendons. They are rarely 
tender; they are found most commonly upon the back of the elbow over the malleoli and at the margin of the patella. They may also be found on the head, especially along the superior curved line of the occiput, the temporal ridge, extensor and flexor surfaces of hands, extensors of feet, vertebral spines, spine of scapula and crista ilii. Other observers have not noted the nodules so frequently as Cheadle. Cantley regarded them as not common, not affecting the prognosis. However, as they may be easily missed, unless carefully looked for, I agree with Cheadle as to their significance and importance and feel sure that their presence probably points to much fibrous change going on in the cardiac valves and the pericardium. They are more commonly associated with the slowly progressive cases than with those acutely rapid and fatal ones. Dr Barlow and Dr Warner recognised their serious import. In 27 cases with nodules there was organic heart disease, in eight pericarditis, in twelve progressive valve disease, and eight were fatal in spite of all treatment. Dr Money found nodules in half the cases of rheumatism in which well marked heart disease occurred. Cheadle's experience is in accord with these observations. The nodules may occur as the sole manifestation, or along with others of the series, but especially with endocarditis, pericarditis and chorea. 
Pleurisy:

A rheumatic pleurisy is now accepted. This may be the sole manifestation, and then not recognised as rheumatic, but with other signs of rhoumatism present, one cannot fail to see the relationship. In one of my cases, Horace Adshead, there was pleurisy with pleuro-pneumonia two or three times. Many of the broncho-pneumonias we meet with in children are probably rheumatic, and $I$ think those soon clear up and are not the long cases with varying ranges of temperature that we sometimes see in other broncho-penumonias.

Endocarditis:

This is the most important of all the manifestations of rheumatism in the child because of its disastrous results, on the condition of the heart valves. In the child it may be the primary manifestation, may occur alone, or along with any of the other members of the series. It is especially important because it may begin in a mild form and may not be suspected while all the time slowly but surely much damage is being done. It is in cases where there may have been some mild passing arthritis, which is not recognised at the time as rheumatic and where the heart is not examined that a slow subacute progressive endocarditis may be 
32 .

doing its deadly work. Severe attacks of articular rheumatism are rare in young children, still children are twice as liable to cardiac inflammation as adults.

Dunn gives a detailed study of a number of consecutive cases healed in the wards of the Children's Hospital. Every case of arthritis, endocarditis and pericarditis admitted to the hospital for a considerable period, somewhat over five years, was included in the study. In this entire course of time only five cases were obviously due to other causes than the rheumatic infection. 300 cases occurred in which no other cause was found. He divided the 300 cases thus:-

(1) With arthritis on admission, 102 cases.

(2) With endocarditis only on admission, 140 cases.

(3) With pericarditis on admission, 58 cases.

These cases show the much greater frequency of cardiac manifestations in children as compared with the articular. Of the 102 cases with arthritis on admission, 85 cases showed signs of valvular endocarditis, and but seventeen children left the hospital with an apparently normal heart. Thus $83 \%$ of the arthritis cases developed signs of - endocarditis. 
Of the 300 cases, 281 showed at some time signs of endocarditis, and 19, none of the 140 cases with endocarditis only on admission, the mitral valve was the most commonly affected. In fact, it was affected in every case but one. Mitral insufficiency alone existed in 70 cases: mitral stenosis alone in one case, aortic insufficiency alone in one case. Mitral insufficiency and stenosis existed in 56 cases. Aortic and mitral insufficiency existed in seven cases; aortic and mitral insufficiency,mitral stenosis existed in five cases.

Dr West's statistics give $60 \%$ of organic valvular disease as rheumatic: M. Roger, 78\%: M. Cadet de Gassecourt 81\%: Goodhart's 62\%: Cheadle's $79 \%$.

Endocarditis in the child may at first show no symptoms beyond some quickening of the pulse or some irregularity of the heart action. In fact, the signs of it may be slight and vague and very easily missed; later, however, changes in the character of the sounds attract notice, the most common is a soft systolic murmur at the apex with accentuation of the pulmonary second sound. Although as a rule endocarditis in children is often insidious and of a subacute nature, occasionally it proceeds with great rapidity. The temperature is rarely high, generally $100^{\circ}-1010$, and often a progressive 
34.

disastrous endocarditis may be going on with a temperature of $99^{\circ}$ or at times normal. The disease progresses with practically no pain: there may be slight dyspnoea. All the valves may become affected but the mitral most commonly and the most common lesion is mitral regurgitation. In fact, throughout the disease there may be no subjective symptoms. With so little to attract one's attention to the condition, one is impressed with the importance of not failing to examine the heart in all cases in children. Endocarditis, rheumatism and chorea all probably own the same cause; and the endocarditis of chorea is now accepted as rheumatic.

\section{Pericarditis:}

The rheumatic poison is recognised now as the most common cause of pericarditis. In the child this manifestation of the rheumatic state is especially important because not only of its crippling effect on the heart, but because of its often fatal result. Like endocarditis it may occur alone, or with others of the series. It may occur early, but as a rule it is a late affection, following on endocarditis.

In the adult pericarditis is often accompanied by serous effusion, and makes itself especially manifest by the pressure symptoms of the effusion. The affected person then shows signs of great distross, with 
35.

much pain, dyspnoea and rapid muffled action of the heart. Although in the child we may meet with a similar condition this is not the rule. Rather do we find the pericarditis runing a subacute insidious and progressive course, disappearing only to recur later, and there is all the time a slow fibrous thickening going on, the two surfaces becoming adherent, when in fatal cases the heart may be seen on post mortem to be enclosed in a tightly fitting fibrous sac.

In the child the pericarditis develops slowly, and at first there may be no signs to mark its occurrence, unless a slight rise of temperature. In a few days, however, we note the expression of the child alters, it becomes anxious looking, and the child seems more distressed with slight pain in the praecordial region, and dyspnoea. This dyspnoea does not necessarily depend on effusion, rather it may be the dread of breathing, or it may possibly be due to myocarditis. There is often pain on pressure over the heart. The pulse quickens to 120 - 130, and the temperature varies usually from $97^{\circ}-100^{\circ}-101^{\circ}$. The quickened pulse in relation to the temperature, is always suggestive of pericarditis, especially if this follows immediately on the endocarditis. When there is endocarditis alone, we may see the child lying happy 
with a cheerful expression, in marked contrast to the ehanged appearance when pericarditis occurs. A slight pericardial rill may be heard at the beginning which disappears when effusion takes place. The anaemia becomes very pronounced. This I think is especially noticeable. The area of cardiac dulness increases, even when the effusion subsides, due, probably, to thickening of the pericardium or possibly to dilatation, as pointed out by Lees and Poynton. The pulse keeps quick, in spite of cardiac tonics. The pericarditis picture is one not to be forgotten once seen. The course may be steadily downward from the outset, the patient becoming more anaemic, the heart more feeble and the patient dies from exhaustion and heart failure; or the symptoms may subside to recur again, and the course of the disease may run into years. The important point to note is that pericarditis may occur primarily, without arthritis or other manifestation of the rheumatic disease, and that it may be present without showing any very definite symptoms at first. In Dunn's 300 cases there were 30 cases in which pericarditis alone or pericarditis with endocarditis were the sole manifestations of infection. The fever persists much longer in pericarditis than in endocarditis, and much longer in endocarditis than in arthritis cases. 
Both in endocarditis and in pericarditis there is a marked contrast between the course of these conditions when due to rheumatism and when due to some septic condition. In the septic cases we have the chills, the irregular fever, a high widely varying chart, and it may be evidences of suppuration at various points, while in the rheumatic cases we get a regular temperature chart and moderate fever, and no other symptoms except the cardiac.

In addition to affecting the endocardium and pericardium, the rheumatic poison may affect the myocardium, setting up a myocarditis. Is this myocarditis the cause of Dilatation of the heart? It may be, and probably is one of the causes of yielding of the heart muscle in the later stages of cardiac cases, apart from valvular lesions. Dr Lees and Poynton, however, have drawn attention, in a valuable paper read before the Medical Chirurgical Society, to the occurrence of dilatation of the heart without the presence of endocarditis and pericarditis. They believe that dilatation of the heart does occur in acute rheumatism, that it may be marked even when there is only slight pyrexia and but little arthritis and with no modification of the cardiac auscultatory phenomena, beyond slight alteration in character of heart sounds. This they think is due to the action of the toxine in the 
heart muscle and comparable to the dilatation so often seen in influenza cases. Poynton found the occurrence of such dilatation in chorea cases, thus forming another link in the rheumatic chain of origin of chorea. I agree with both these observers as to the possibility of such occurrence, and think that many cases of cardiac failure are not due to breaking down of compensation from over exertion but rather due to yielding of the muscle, through weakening from fresh infections. Such dilatation supports my belief of a mild infection going on without any other sign than anaemia, while if the heart in many of these cases was carefully examined, slight dilatation might be found to be present. Such dilatation may explain many of the causes of sudden death from cardiac failure in these cases convalescent, or supposed to be convalescing. Nothing bears out the importance of examining the heart in children in what are thought to be slight and trifling ailments, than what we know of the mild, insidious progressive ard disastrous nature of cardiac affections in the rheumatic disease of childhood.

\section{Diagnosis:}

Careful investigation into the family history of children with slight feverish attacks, even without arthritis, is important. If we get a child 
with slight temperature and signs of cardiac affection alone, the probability is that such affection is rheumatic;if there is arthritis, then the probability is greater. A history of previous attacks still further increases the probability. Previous attacks of sore throat or chorea or concurrent attacks all strengthen the diagnosis. One should be on the look out for any one of the manifestations. To me the anaemia which I have seen so often in these cases often leads to a diagnosis, as children, especially in the country, are not usually anaemic, unless where there are signs of some other plainly recognisable cause, such as fault in dietary or some wasting disease.

\section{Prognosis:}

The prognosis is much more grave in the case of children, as the insidious character of the cardiac affection leads it to be overlooked; the arthritis, being very mild or not noticed by the parents, allows a disastrous heart affection to progress and do imparable damage, before the case is seen by a medical man. Then the tendency to recur, with fresh infection of the heart tisaves, leads in time to fatal damage. It has been well said that rheumatic children grow into heart disease. In mild cases or in those recognised early 
and carefully protected throughout the remainder of childhood, the cases may recover, and the valvular lesion be compensated. The articular cases, without cardiac symptoms, are much more favourable, but these also must be carefully guarded from all evil influences. In Dunn's series of 300 cases, 55 died in hospital, i.e. 18.33\%. He states that in a case of rheumatic fever in early life, the chances of death may be as high as one in five. Cardiac failure is usually the cause of death in all cases, but we rarely see the dropsical condition met with in cardiac failure in adults.

\section{Treatment:}

Since rheumatic fover is now known to be one of the most common and most damaging of all diseases, it is of the first importance to prevent its occurrence if possible, and to aid this, it is important to study the predisposing causes ; the influence of climate, cold and damp, the influence of houses, overcrowding etc. It is important to study epidemic outbreaks and cases of apparent contagion. Children of rheumatic stock should be especially guarded from chills, should be well clothed and well fed, and should never be allowed to fatigue themselves. 
Since damage to the heart is the principal condition to be guarded against, one must be on the look out for any insidious, mild endocarditis or pericarditis, and since we now know that such an attack may arise apart from amy of the others of the rhoumatic series, with probably only a very slight feverishness, it behoves us then to examine the condition of the heart carefully, not only in every case where there is the mildest articular affection or other rheumatic affection such as a stiff neck, knee, wrist or ankle, a tonsillitis, nodules or chorea, but in every pyrexial condition in a child - in fact, it is well to examine the heart always. Once we suspect the presence of rheumatism, rest in bed should be enforced, even in the slightest cases and even if no cardiac disturbance is detectable. The child should be clothed in flannel and placed between blankets, if possible, in a bedroom exposed to the sun. Dr Byrom Bramwell in his clinical studies, shows us how important in these cases is sunlight. The diet should consist principally of milk. I give nothing but milk with barley soda or hot water, until the temperature is normal. The child should be kept in bed for at least ten days after the temperature has returned to normal. Where there is a murmur present and whether this is thought to be functional or organic, 
long rest in bed for six or eight weeks at least should be ordered, as even organic murmurs with such rest may disappear.

\section{Medical:}

When one considers the medical treatment, it seems to resolve itself into, alkaline alone, salicylates alone, or a combination. It is now generally accepted since McLagan introduced salicin that the salicylates are specific in acute rheumatism. Some such as Lees give very large doses : others fear to do so because of the action of the drug on the heart, but the rheumatic poison is more damaging to the heart than the salicylates are. I have found in a child of ten years of age, doses of 10 grains of salicylate of soda with 15 grains of sodae or Potas. Bicarbonate repeated every two hours to act well. I keep up this dosage for three days, even although the temperature has returned to normal, and the joint pain gone; the fourth day I give the same doses every three hours; the fifth day every four, and so on, and at the end of the week I give it every six and keep this up for a fortnight or three weeks. The important point is to get the system quickly saturated with the salicylate, as well shown by Professor Stockman. He, says: "The most probable explanation of the rapid 
subsidence of the swelling and pain is that locally in the joint an antitoxic or antibacterial substance is formed which powerfully antagonises the poisonous agent and does it so rapidly that the joints are seldom injured. The closed cavity of the joint probably favours the accumulation of the antibody until it reaches a highly active degree of concentration. This view is supported by the circumstance that while the fibrous tissues of the joints, tendons and aponeuroses are also very often the seat of rheumatism, it is well recognised that in these places the inflammation is usually very persistent and apt to leave behind permanent fibrous thickenings, and this, I think, is owing to the antibody being in much less quantity in the body serum than in the joint effusion." "The explanation of the rapid action of the sodium salicylate on the pain and swelling of joints is that it is excreted into the joint cavity from the blood in comparatively large amount as can be readily shown by its detection in the fluid withdrawn by means of a hypodermic syringe. On the other hand, colonies of microbes settled deep in the fibrous tissues or in the valves of the heart are more or less sheltered from the action of the salicylate and are probably scarcely reached by it or if reached, the solution in the serum is too dilute 
to exert much action." He further suggests this as the explanation why valvular rheumatic infections and infections of the fibrous tissues generally are not nearly so amenable to the action of the salicylates as the joints are. With this I entirely agree and think that these fibrous infections are those where Quinine is sometimes useful. With the above adnirable explanation, we must push the salicylates for days, even when the temperature is normal as nothing is more common than relapses every third or fourth day if the salicylates are not kept up.

In cases of pericarditis, an ice-bag as suggested by Dr Less, or warm poultices, are beneficial or sinall mustard applications as suggested by Dr Caton, in elder children. If cardiac failure threatens, we must depend on strychnine, digitalis or strophanthin. For pain, an opiate is often required, although in pericarditis I found unless I increased the salicylates this did not relieve much.

The important point, I again restate, is to give sufficient doses of the salicylate early and keep up the dosage sufficiently long. I also believe in a course of salicylates, twice a year at least, in the intervals for rheumatic children. When convalescent, a return to fish, whitemeat and faunaceous foods is allowable; not too much of 
the faunaceous, however. Then one must caution the parents against any overexertion when heart is damaged, as we may thus have compensation broken down, although as mentioned before, those frequent break-downs of compensation may not be due only to overexertion but may mean fresh infection. All stomach troubles should be carefully attended to and the action of the bowels carefully regulated, and so keep the body in the best state to resist any infection. 
The undermentioned have been consulted by me:-

1. "Rheunatism and its allies in childhood", Barlow, British Medical Journal, 1883, Vol.I.I. 509.

2. Harveian Lectures, Cheadle, 1889.

3. British Medical Journal, October 13, 1906. "Blackadder on Rheumatism in children".

4. British Medical Journal, May 14th, 1904, Poynton, "Remarks on infective nature of rheumatic fever." Fatal case.

5. Poynton and Paine. Lancet, Sept. 22-29, 1900.

6. Beattie. British Medical Journal, Decr. 1906.

7. British Medical Journal, 29th Augt. 1903,

"Lees". Discussion on the pathology and treatment of chorea.

8. British Medical Journal, June 23, 1906. Chorea, considered as cerebral rheumatism, Sir Dyce Duckworth.

9. Journal of Medical Association of America, February 9, 1907. Articles by snyder and Dunn.

10. British Medical Journal, 24th Nov. 1906,

"A Clinical. Lecture on the action of salicylates in acute rheumatism" by Professor Stockman.

11. Clinical Studies, Bryom Bramwell, Vol.I. Rheumatic fever and endocarditis.

12. Transaction of Royal Medical \& Chirurgical Societies, Lees, Poynton. "On dilatation of heart in acute rheumatism and chorea" June 1898.

13. Cheadle, Acute Rhoumatism of Childhood, in Allbutt's System of Medicine, Vol.III.

14. R. Russell on Chorea in Allbutt's System of Medicine, Vol.VII. 
15. Oslers. Medicine. Chorea.

36. Archiv. f. psychiatric. 37, p.86, 1903.

Beitrag. zur. Pathologischen Anatomic der chorea minor. Von Dr Carl Hudovering. 